BLS 35, No 1 2009. DOI: http://dx.doi.org/10.3765/bls.v35i1.3601

(published by the Berkeley Linguistics Society and the Linguistic Society of America)

\title{
Pitch Height vs. Contour in Tonal Perception in Fuzhou
}

\author{
CATHRYN DONOHUE \\ Australian National University
}

\section{Introduction}

The goal of this paper is to investigate the degree to which the slight tonal contour of the three phonologically level tones plays a role in the perception of these tones. First I introduce the tones in Fuzhou, identifying the tones that are under study. Next I outline the method used to create the synthetic tones as well as the perception study. Finally I present the results and discuss the significance of the F0 contour in Fuzhou.

\section{Fuzhou}

Fuzhou, a Min dialect spoken in north-eastern Fujian province, China, has seven citation tones. A detailed description of these tones based on auditory impressions is given in Figure 1 (Donohue, to appear).

Figure 1. A description of the citation tones in Fuzhou

Tone 1 High level with a slight rise

Tone 2 Mid level with a slight fall

Tone 3 Low level with a slight fall

Tone 4 Low rising stopped tone (final glottal stop)

Tone 5 High fall, starting higher than the high level tone

Tone 6 Low rise-fall

Tone 7 High stopped tone (final glottal stop), notably shorter than the others

Earlier phonological work addressing Fuzhou has typically assumed that tones 1-3 are level tones (e.g. Yip 1980, Chan 1985, Jiang-King 1996, Donohue, to appear). While they are the most level tones in the set, an acoustic normalization nonetheless revealed a consistent rise for tone 1 and a consistent and similar, falling contour for tones 2 and 3 (Donohue 1992a, b).

In order to examine the role of contour vs "pitch" (F0) height, I focus on tones 1 and 2, which are the closest two 'level' tones that illustrate this difference in contour. 


\section{Cathryn Donohue}

\section{Experimental Methodology}

In an earlier study, Donohue (1992a) presents a description of the quantified tones, normalizing across 4 speakers. The starting point for this experiment was a set of tokens from a single speaker that most closely represented the mean normalized set of F0 contours.

In order to investigate the role of contour on the perception of these two 'level' tones, I synthesized tokens whose contour matched that of the original token, but modifying the overall F0 height as measured at the point of onset.

\subsection{Synthetic Tones}

I created tokens in Praat by modifying the F0 such that for each of tones 1 and 2, there would be synthetic tokens where the F0 would start at the midway point between the onset of the two tones, at three-quarters of the distances towards the other tone and at the point of onset of the other tone. That is, six synthetic tones were created and included among the non-modified tokens presented to the listeners. These tones are illustrated below in figures $2 \mathrm{a}$ and $2 \mathrm{~b}$.

Figure 2a. The synthetic tones (dashed) for tone 1 and natural tones 1 and 2 .

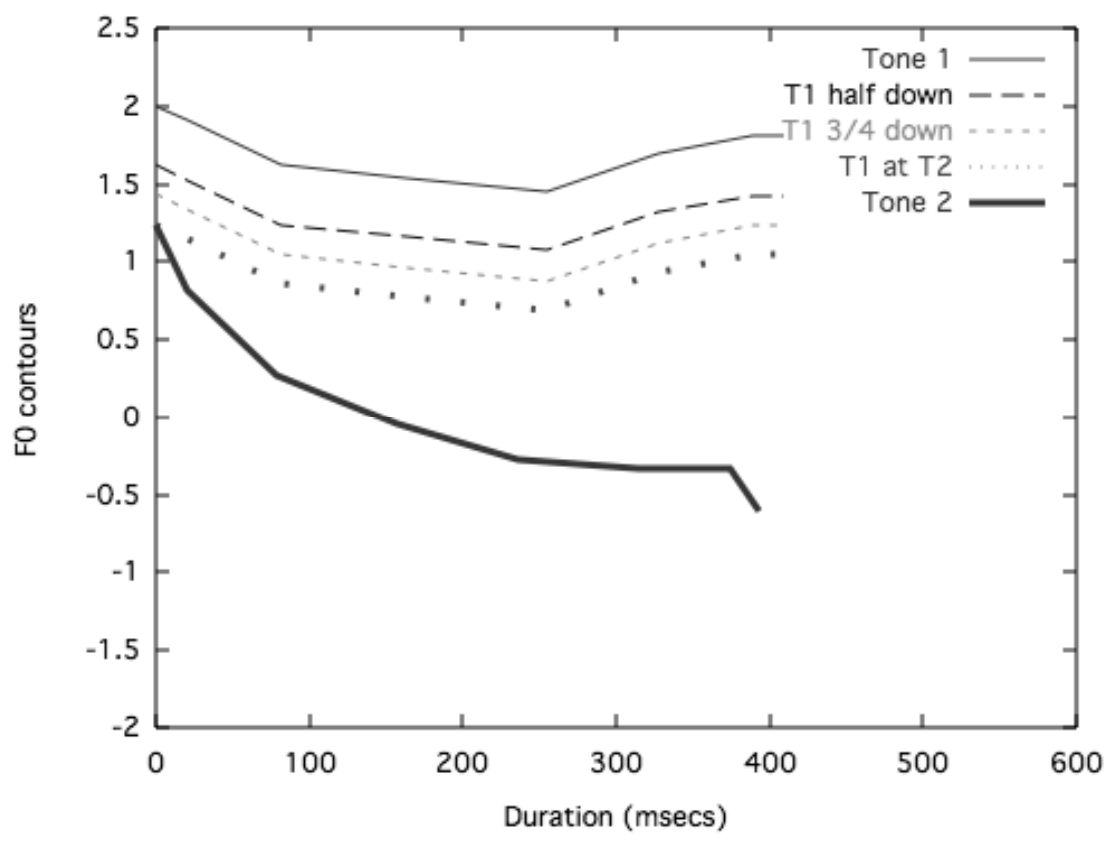


Figure $2 \mathrm{~b}$. The synthetic tones (dashed) for tone 2 and natural tones 1 and 2 .

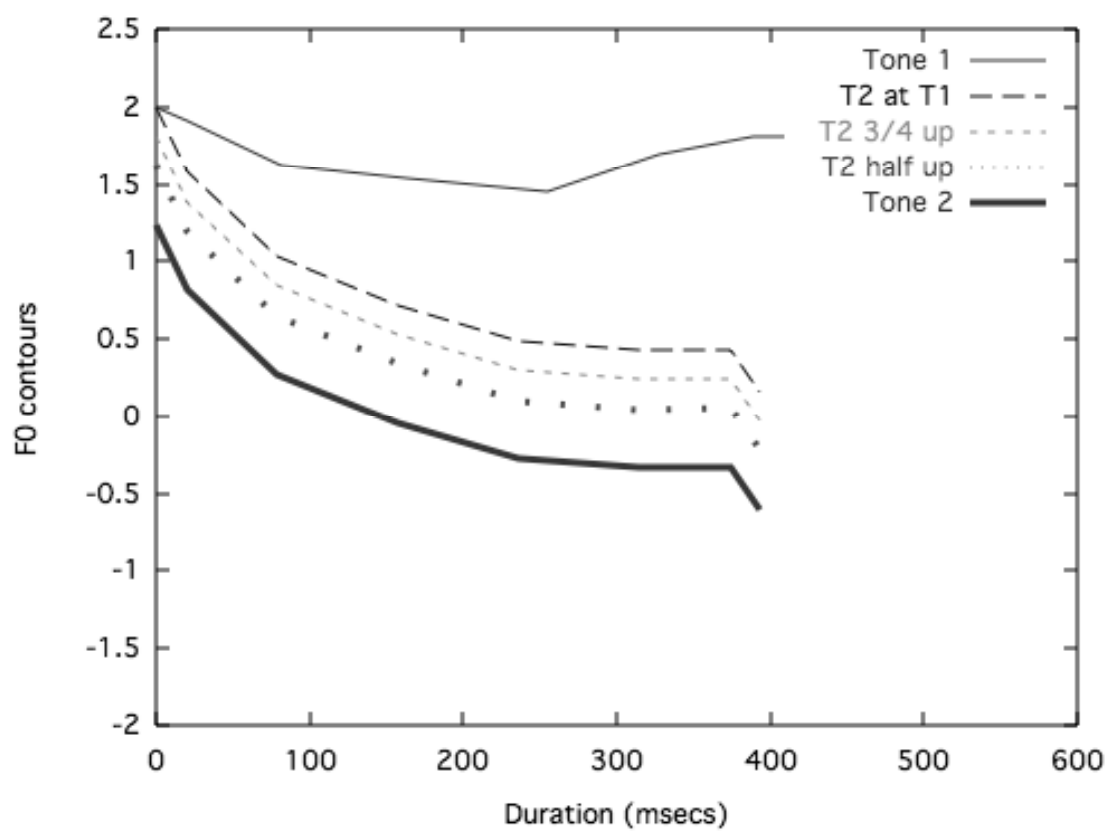

\subsection{Perception Study}

The data consisted 41 tokens, comprised of both the (target) synthetic tokens interspersed with natural tokens (of all seven citation tones). 13 native speakers of Fuzhou were given a list of seven characters and were asked to indicate when they heard a word from that list. The listeners were presented the tokens in a random order and listened to the whole set twice.

\section{Results of the Experiment}

The key result is the analysis of the two synthetic tones starting at the midway point between the two F0 onsets: With the same "pitch height," these tones differ only in their contour.

In order to determine the role of contour, the working hypothesis we can establish is that proportion of times, $p_{0}$, that a listener would choose the tone with the matching contour (tone 1 or tone 2 ) if contour did not matter would be 0.5 .

To determine whether the difference between the observed proportion $\mathrm{p}$ and the assumed proportion $p_{0}$ is statistically significant, we can apply a Z-test for proportion. The test statistic is given in (1).

$$
\mathrm{Z}=\frac{\left|p-p_{0}\right|-\frac{1}{2 n}}{\sqrt{\frac{p_{0}\left(1-p_{0}\right)}{n}}}
$$




\section{Cathryn Donohue}

At the midway point there are 52 data points, 45 of which correctly identify the tone. Thus, $\mathrm{n}=52, \mathrm{p}=45 / 52=0.865$. This yields a $\mathrm{Z}=5.13$. The critical value $\mathrm{Z}_{0.001}$ is 3.29 . We can therefore reject, with $99.9 \%$ confidence, the hypothesis that there is no difference in the proportions (i.e. that contour plays no role in tone identification). That is, contour does play a role in tonal identification for the phonologically level tones in Fuzhou.

When F0 is moved three-quarters the distance towards the other tone: The tones were correctly identified $83 \%$ of the time. Thus the F0 height has an effect, but it is a small one. Finally, when the tones are moved to the same F0 onset height as the other tone: The tones are still correctly classified $81 \%$ of the time.

\section{Concluding Remarks}

This study has shown that the phonologically 'level' tones crucially rely on both the slight contour and phonation change for their perception. In future work I plan to investigate the role of F0 height and its interaction with phonation in the perception of these tones as well as the concomitant vowel changes (avoided in this study) and the possible influence of duration on the perception of tones in Fuzhou.

\section{References}

Chan, Marjorie. 1985. Fuzhou phonology: A non-linear analysis of tone and stress. Ph.D. diss., Department of Linguistics, University of Washington.

Donohue, Cathryn. 1992a. The phonetics and phonology of Fuzhou tones. Honours thesis, Australian National University.

Donohue, Cathryn. 1992b. The phonetics of register in the Fuzhou dialect of Chinese. In Proceedings of the Fourth Australian International Conference on Speech Science and Technology, 602-607. Brisbane, University of Queensland Press.

Donohue, Cathryn. To appear. Tones and vowels in Fuzhou. In Proceedings of the $33^{\text {rd }}$ Annual Meeting of the Berkeley Linguistics Society. Berkeley, CA: Berkeley Linguistics Society.

Jiang-King, Ping. 1996. An optimality account of tone-vowel interaction in northern Min. Ph.D. diss., Department of Linguistics, University of British Columbia.

Yip, Moira. 1980. The tonal phonology of Chinese. Ph.D. diss., Massachusetts Institute of Technology.

Department of Linguistics

Research School of Pacific \& Asian Studies

Australian National University

Canberra ACT 0200

Australia

cathryn@donohue.cc 